\title{
30 Jahre Mauerfall und die Rückkehr der Appeasement Politik
}

Citation for published version (APA):

Brand, H. (2019). 30 Jahre Mauerfall und die Rückkehr der Appeasement Politik. Das Gesundheitswesen, 81(11), 860-861. https://doi.org/10.1055/a-1023-8941

Document status and date:

Published: 01/11/2019

DOI:

10.1055/a-1023-8941

Document Version:

Publisher's PDF, also known as Version of record

Document license:

Taverne

Please check the document version of this publication:

- A submitted manuscript is the version of the article upon submission and before peer-review. There can be important differences between the submitted version and the official published version of record.

People interested in the research are advised to contact the author for the final version of the publication, or visit the DOI to the publisher's website.

- The final author version and the galley proof are versions of the publication after peer review.

- The final published version features the final layout of the paper including the volume, issue and page numbers.

Link to publication

\footnotetext{
General rights rights.

- You may freely distribute the URL identifying the publication in the public portal. please follow below link for the End User Agreement:

www.umlib.nl/taverne-license

Take down policy

If you believe that this document breaches copyright please contact us at:

repository@maastrichtuniversity.nl

providing details and we will investigate your claim.
}

Copyright and moral rights for the publications made accessible in the public portal are retained by the authors and/or other copyright owners and it is a condition of accessing publications that users recognise and abide by the legal requirements associated with these

- Users may download and print one copy of any publication from the public portal for the purpose of private study or research.

- You may not further distribute the material or use it for any profit-making activity or commercial gain

If the publication is distributed under the terms of Article $25 \mathrm{fa}$ of the Dutch Copyright Act, indicated by the "Taverne" license above, 


\section{Jahre Mauerfall und die Rückkehr der Appeasement Politik}

Vor 30 Jahren fiel der Eiserne Vorhang und nächstes Jahr wird dieses Jubiläum in Bezug auf die deutsche Wiedervereinigung begangen. Nach 20 Jahren war die Schere in der Lebenserwartung zwischen Ost- und Westdeutschland weitgehend geschlossen und wir sehen jetzt einen von Nord-Ost nach Süd-West verlaufenden Gradienten. Eine um Kaufkraft bereinigte Analyse zeigt, dass die 10 am weitesten abgehängten Kommunen mittlerweile in West- und nicht in Ostdeutschland liegen.

Wenn Koreaner uns fragen, was sie für sich aus der deutschen Wiedervereinigung lernen können, kann man nur antworten, dass es dauert, Geld kostet und man nicht alles für alle richtig machen kann.

So findet sich in Ostdeutschland ein Gefühl des Abgehängt-Seins und einer nicht anerkannten Lebensleistung, die auch zur Wahl von populistischen Parteien beigetragen hat.

Den Trend hin zu Parteien, die einfache Lösungen auf komplexe Fragestellungen liefern, sieht man allerdings in ganz Europa, weshalb dies kein spezielles deutsches Problem ist.

Viele Menschen sind beruhigt, wenn ihre Protestwahl Parteien in das Parlament bringt, die populistisch agieren. „Sie sind ja nicht in der Regierung, sondern nur in der Opposition“, wird oft herabmildernd gesagt. Gerade dies ist trügerisch! Parteien, die in Parlamenten sitzen, egal ob auf kommunaler, Landesoder Bundesebene, haben einen - oft auch ungeschriebenen - Anspruch auf gewisse Rechte und Vertretung in Gremien. Es sei hier an die Besetzung der Position der Bundestagsvizepräsidenten erinnert. Diese Diskussion war und ist sehr medienwirksam, die wahre Problematik - auch für die öffentliche Gesundheit - liegt aber eher in anderen Bereichen.

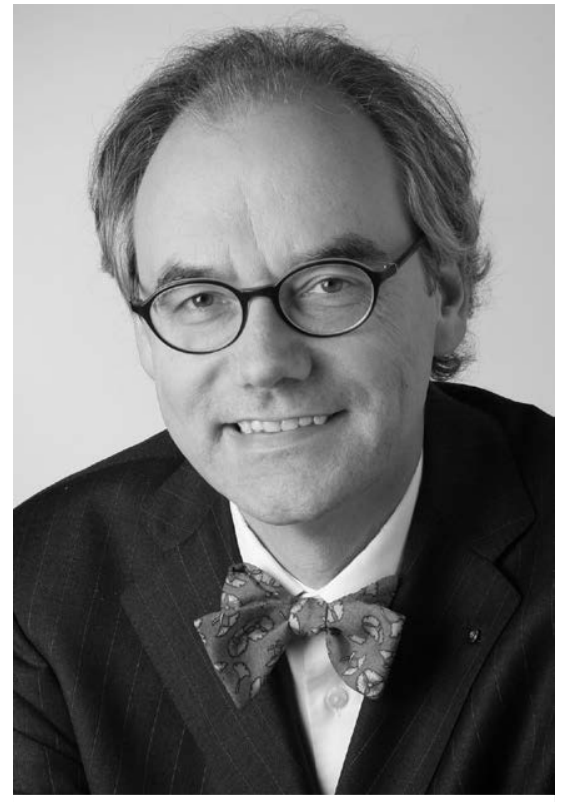

Helmut Brand ist Jean Monnet Professor in European Public Health an der Universität Maastricht. In seinem Kommentar „Mail aus Maastricht" greift er aktuelle Themen der europäischen Gesundheitspolitik auf.

Wenn auf kommunaler Ebene Aufsichtsratsposten von kommunalen Betrieben besetzt werden, wird man an diesen Parteien auf Dauer nicht vorbeikommen. Desgleichen gilt für die Landes- und Bundesebene. Es werden dann auch Förderanträge oder strategische Ausrichtungen hinterfragt werden. Das beliebte Instrument der kleinen und großen Parlamentsanfrage wird schon jetzt sehr häufig benutzt, um - oft vermeintliche - Missstände anzuprangern. Es sollen hier nicht die Rechte von Parlamentariern in Frage gestellt werden, wir müssen uns aber bewusst sein, dass dies auch immer politische (Hilfs-) Mittel sind.

Wie reagieren die Mitarbeiter von Verwaltungen - also auch von Gesundheitsfachverwaltungen - auf diesen Trend? Oberflächig betrachtet wird weiter alles korrekt rechtsstaatlich abgearbeitet.
Mitarbeiter der Fachverwaltung bearbeiten politische Anfragen. Dies wird meistens nicht gern getan, da sie die Routine stören, Zeitdruck erzeugen und damit von „wichtigeren“ Arbeiten abhalten. Wenn es dann noch um Fragen geht, die politisch gemeint und in neutrale Schachverhalte verpackt werden, dann wird es schwierig. (Weiß die Landesregierung wie viele deutsche Staatsbürger von Flüchtlingen mit Tuberkulose angesteckt wurden?)

Wird dann auch zusätzlich die Arbeit der Fachbehörde hinterfragt, in dem man z. B. die Vergabe von Gutachten und Aufträgen mit einer vermeintlichen Gesinnung der Auftragnehmer verbindet, dann wird es unangenehm.

Fragen, die etablierten Verfahrensweisen in Frage stellten, gab es allerdings schon als z. B. ökologische Parteien in die Parlamente einzogen. Dies hat zum einen für einen gewissen gesunden frischen Wind in den Parlamenten gesorgt. Zum anderen wurden aber auch manche fachpolitischen Entscheidungen in eine neue Richtung gedrängt.

Mittlerweile sind ökologische Parteien im Mainstream angekommen und haben eine große Wählerschaft. Dazu gehört auch, dass jetzt Mitarbeiter in den administrativen Fachabteilungen zu finden sind, die Mitglieder dieser Partei sind - was völlig in Ordnung ist.

Das heißt, in jeder Fachverwaltung gibt es auch Anhänger des Populismus, was nur die Verteilung in der Bevölkerung widerspiegelt. Wie reagiert die Fachverwaltung auf dieses Geschehen? Wird sie sich noch trauen, den Antrag zu freiwilligen kommunalen Leistungen zur Unterstützung alternativer Lebensformen im Vorfeld zu unterstützen oder gleich versuchen, ihn abzuwimmeln? Wird sich eine Selbstzensur bei der Vergabe von Gutachten und Aufträgen entwickeln, die versucht, die unliebsamen kleinen und großen Anfragen zu vermeiden? Wird man 
noch Initiativen zur gesundheitlichen Versorgung von Flüchtlingen im Rahmen freiwilliger Leistungen starten?

Es ist jetzt - eigentlich wie immer - Zivilcourage der Fachverwaltungen gefordert. Es geht bei Fachentscheidungen eben nicht darum, das mögliche Aufschreien von Vertretern populistischer Parteien vorweg zu nehmen. Mitarbeiter öffentlicher Verwaltungen sind unkündbar. Nicht, damit sie sich vor Parteiideologie wegducken, sondern damit sie dem Gemeinwohl dienen. Und dies auch unter dem Risiko eines möglichen Mobbings durch Vorgesetzte oder einer verspäteten Beförderung.

All dies Beschriebene ist eine europaweite Problematik. Vertreter populistischer Par- teien sind mittlerweile an Regierungen beteiligt und stellen auch Staatschefs. Die neue Kommissionspräsidentin wurde einstimmig - also auch mit den Stimmen populistischer Regierungschefs - im Europäischen Rat gewählt. Man kann nur hoffen, dass wir keine „Miss Chamberlain“ sehen werden, die sich im Gegenzug zu ihrer Wahl einer neuen Appeasement Politik hingibt. Die Diskussion um die Besetzung der Kommissare zeigt, dass die Europaparlamentarier, nachdem sie bei dem Prozess der Besetzung der Leitung der Kommission keine gute Figur abgegeben haben, jetzt aufgewacht sind.

Die Kommissionspräsidentin hat gute, primär nicht ideologische Ideen in den Aufgabenkatalog der neuen Gesundheitskommissarin
Stella Kyriakides geschrieben. Gleichzeitig aber hat die Formulierung der „Verteidigung der europäischen Werte“ in ihrer Parlamentsrede doch zu einigen Irritationen geführt. Das Räderwerk läuft also noch nicht rund. Wir sollten, nein, wir müssen deshalb die Kommission in einer gegen populistisches Gedankengut immunisierten Arbeit unterstützen. Und dies nicht nur in DG Sante, der europäischen Gesundheitsfachverwaltung, sondern in allen Politikbereichen, da der Ansatz von „Health in all Policies“ Bestandteil der europäischen Verträge ist.

Helmut Brand, Universität Maastricht 